\title{
$\omega$-Calderón-Zygmund operators
}

by

SIJUE WU (Evanston, III.)

Abstract. We prove a $T 1$ theorem and develop a version of Calderón-Zygmund theory for $\omega$-CZO when $\omega \in A_{\infty}$.

1. Introduction. Let $T$ be a linear operator mapping test functions on $\mathbb{R}^{n}$ continuously into distributions and with an associated kernel $K(x, y)$, $x \neq y$ (in the sense that $\langle T f, g\rangle=\iint g(x) K(x, y) f(y) d x d y$ whenever $f$ and $g$ are test functions with compact support). Let $\omega$ be an $A_{\infty}$ weight with critical exponent $p_{0}$, and set $\omega_{t}(x)=t^{-n} \int_{|x-y|<t} \omega(y) d y$. We say that $K(x, y)$ satisfies $\omega$-standard estimates if for some $\varepsilon>0$,

$$
|K(x, y)| \leq C \omega_{|x-y|}(x) \frac{1}{|x-y|^{n}}
$$

and

$$
\begin{aligned}
& \left|K(x, y)-T_{x^{\prime}} K(x, y)\right| \leq C \omega_{\left|x-x^{\prime}\right|}(x) \frac{\left|x-x^{\prime}\right|^{\varepsilon}}{|x-y|^{n+\varepsilon}} \\
& \quad \text { if }\left|x-x^{\prime}\right|<\frac{1}{2}|x-y|, \\
& \left|K(x, y)-T_{y^{\prime}} K(x, y)\right| \leq C \omega_{\left|y-y^{\prime}\right|}(y) \frac{\left|y-y^{\prime}\right|^{\varepsilon}}{|x-y|^{n+\varepsilon}} \\
& \quad \text { if }\left|y-y^{\prime}\right|<\frac{1}{2}|x-y|,
\end{aligned}
$$

where

$$
\begin{aligned}
T_{x^{\prime}} K(x, y)= & K\left(x^{\prime}, y\right)+\left(\left(x-x^{\prime}\right) \cdot D_{x}\right) K\left(x^{\prime}, y\right) \\
& +\frac{1}{2 !}\left(\left(x-x^{\prime}\right) \cdot D_{x}\right)^{2} K\left(x^{\prime}, y\right) \\
& +\ldots+\frac{1}{N !}\left(\left(x-x^{\prime}\right) \cdot D_{x}\right)^{N} K\left(x^{\prime}, y\right)
\end{aligned}
$$

1991 Mathematics Subject Classification: Primary 42B20. 
and

$$
\begin{aligned}
T_{y^{\prime}} K(x, y)= & K\left(x, y^{\prime}\right)+\left(\left(y-y^{\prime}\right) \cdot D_{y}\right) K\left(x, y^{\prime}\right) \\
& +\frac{1}{2 !}\left(\left(y-y^{\prime}\right) \cdot D_{y}\right)^{2} K\left(x, y^{\prime}\right) \\
& +\ldots+\frac{1}{N !}\left(\left(y-y^{\prime}\right) \cdot D_{y}\right)^{N} K\left(x, y^{\prime}\right)
\end{aligned}
$$

(with $D_{x}=\left(\partial / \partial x_{1}, \ldots, \partial / \partial x_{n}\right)$ and $\left.D_{y}=\left(\partial / \partial y_{1}, \ldots, \partial / \partial y_{n}\right)\right)$ are the Taylor polynomials of degree $N$ of $K(x, y)$ with respect to $x$ (at $x^{\prime}$ ) and $y$ (at $y^{\prime}$ ), respectively, and $N=\left[n\left(p_{0}-1\right)\right]$, where $[a]$ stands for the biggest integer $\leq a$.

An example of such an operator is the fractional integral

$$
I_{\alpha} f(x)= \begin{cases}\int_{\mathbb{R}^{n}}|x-y|^{\alpha-n} f(y) d y & \text { if } \alpha>0 \text { and } \alpha \neq n+2 l, \\ \int_{\mathbb{R}^{n}} y|y|^{\alpha-n-1} f(x-y) d y & \text { if } \alpha>0 \text { and } \alpha=n+2 l,\end{cases}
$$

where $l$ is an integer. In these cases, we can take $\omega=|x|^{\alpha}$.

For the case $\omega \in A_{1}$, a version of the $T 1$ Theorem has been proved and a Calderón-Zygmund theory for $\omega-\mathrm{CZO}$ has been developed in [3]. In this paper, we will give a version of the $T 1$ Theorem for $\omega \in A_{\infty}$ and prove a Calderón-Zygmund theory for $\omega$-CZO which contains the theory in [3] as a special case.

Acknowledgements. This paper is part of my thesis. I would like to express my deep gratitude to my advisor, Prof. Ronald R. Coifman, for proposing the problem and for his advice. I would also like to thank Prof. José García-Cuerva for the very helpful discussions during his visit in Yale.

2. The statement of the $T 1$ Theorem. Let $\omega$ be an $A_{\infty}$ weight with critical exponent $p_{0}$. Suppose $N=\left[n\left(p_{0}-1\right)\right]$. Let $T$ be a continuous linear operator from test functions to distributions. We say that $T$ satisfies the $\omega-W B P$ (weak boundedness property) if for each ball $B$ and any test functions $\phi_{1}, \phi_{2}$ supported in $B$ that satisfy the estimate $\left\|\phi_{i}^{\prime}\right\|_{\infty} \leq|B|^{-1 / n}$ for $i=1,2$ and the cancellation law

$$
\int x^{k} \phi(x) d x=0 \quad \text { for } k=0,1, \ldots, N
$$

where $\phi=\phi_{1}$ or $\phi_{2}$, we have

$$
\left|\left\langle T \phi_{1}, \phi_{2}\right\rangle\right| \leq C \omega(B) .
$$

This will hold if $T$ is associated with an antisymmetric kernel satisfying the first standard estimate (1.1).
The space $B M O_{\omega}=\left(H^{1}(\omega)\right)^{*}$ has been studied in [5]. The definition is, for $\omega \in A_{p}, 1 \leq p<\infty$,

$$
\begin{aligned}
B M O_{\omega}=\left\{f: f / \omega \in L_{\mathrm{loc}}^{p^{\prime}}(\omega) \text { and for every interval } I,\right. & \\
& \left.\left(\omega(I)^{-1} \int_{I}\left|f(x)-P_{I} f(x)\right|^{p^{\prime}} \omega(x)^{1-p^{\prime}} d x\right)^{1 / p^{\prime}} \leq C\right\},
\end{aligned}
$$

where $1 / p+1 / p^{\prime}=1$ and $P_{I} f$ is a polynomial of degree $\leq N$ such that

$$
\int_{I}\left(f(x)-P_{I} f(x)\right) x^{k} d x=0 \quad \text { for } k=0,1, \ldots, N,
$$

and the $B M O_{\omega}$ norm of $f \in B M O_{\omega}$ is the smallest constant $C$ in the inequality defining $B M O_{\omega}$. For more details, see [5]. We also refer to [5] or [6] for the definition of weighted Hardy spaces $H^{p}(\omega)$.

THeOREm 2.1. Suppose $\omega \in A_{\infty}$. Let $T$ be a continuous linear operator from test functions to distributions that is associated with an $\omega$-standard kernel. Then $T: H^{1}(\omega) \rightarrow L^{1}$ iff $T$ satisfies the $\omega$-WBP and T1 and $T^{t} 1$ lie in $B M O_{\omega}$.

A corollary of this theorem is

Corollary 2.2. Same assumptions as in Theorem 2.1. Then $T: H^{1}(\omega)$ $\rightarrow L^{1}$ and $T: L^{\infty} \rightarrow B M O_{\omega}$ iff $T$ satisfies the $\omega-W B P$ and $T 1$ and $T^{t} 1$ lie in $B M O_{\omega}$.

3. The proof of the theorem. In the following, all $C$ 's are constants, and they need not be the same at each appearance. Moreover, $1 / p+1 / p^{\prime}=1$.

The proof of Theorem 2.1 follows the same steps as in [3] and [4]. We prove the sufficiency first. Because $\omega \in A_{\infty}$, we have $\omega \in A_{p}$, for some $2<p<\infty$.

LEmmA 3.1. Suppose $\phi, \psi \in C^{\infty}, \operatorname{supp} \phi, \psi \subseteq\{|x| \leq 1\}, \int x^{k} \psi(x) d x=0$ for $k=0,1, \ldots, N$, and $b \in B M O_{\omega}$. Define $P_{t} f=\phi_{t} * f, Q_{t} f=\psi_{t} * f$, where $\phi_{t}(x)=t^{-n} \phi(x / t)$. Then the kernel of the paraproduct operator

$$
T_{b} f=\int_{0}^{\infty} Q_{t}\left(\left(Q_{t} b\right)\left(P_{t} f\right)\right) \frac{d t}{t}
$$

satisfies the second $\omega$-standard estimate (1.2) and $T_{b}: H^{p^{\prime}}(\omega) \rightarrow L^{p^{\prime}}\left(\omega^{1-p^{\prime}}\right)$.

Proof. The kernel of the operator is

$$
K(x, y)=\int_{0}^{\infty} l_{t}(x, y) \frac{d t}{t}=\int_{0}^{\infty} \int_{\mathbb{R}^{n}} \psi_{t}(x-z) Q_{t} b(z) \phi_{t}(z-y) d z \frac{d t}{t} .
$$


We have

$$
\begin{aligned}
\mid l_{t}(x, y) & -T_{x^{\prime}} l_{t}(x, y) \mid \\
= & \left|\frac{1}{(N+1) !}\left(\left(x-x^{\prime}\right) \cdot D_{x}\right)^{N+1} l_{t}(\xi, y)\right| \\
= & \frac{1}{(N+1) !}\left|\int\left(\left(x-x^{\prime}\right) \cdot D\right)^{N+1} \psi_{t}(\xi-z) Q_{t} b(z) \phi_{t}(z-y) d z\right|
\end{aligned}
$$

for some $\xi=x^{\prime}+\theta\left(x-x^{\prime}\right), 0 \leq \theta \leq 1$, and $\left|x-x^{\prime}\right|<\frac{1}{2}|x-y|$. Because $b \in B M O_{\omega}$ and $\psi_{t} \in H^{1}(\omega)$ (in fact, $\left(\omega_{t}(z)\right)^{-1} \psi_{t}(z-\cdot)$ is a $(1, \infty)$-atom), we have

$$
\left|Q_{t} b(z)\right|=\left|\int \psi_{t}(z-u) b(u) d u\right| \leq\left\|\psi_{t}\right\|_{k^{1}(\omega)}\|b\|_{*}=C_{\psi}\|b\|_{*} \omega_{t}(z)
$$

Then for any $x$ with $|z-x|<t$, we have

$$
\left|Q_{t} b(z)\right| \leq C\|b\|_{*} \omega_{t}(z) \leq C\|b\|_{*} \omega_{t}(x)
$$

and

$$
\left|l_{t}(x, y)-T_{x^{\prime}} l_{t}(x, y)\right| \leq \frac{C_{\phi, \psi}}{(N+1) !}\left|x-x^{\prime}\right|^{N+1} \frac{\omega_{t}(x)}{t^{N+n+1}} \chi_{\{|x-y|<4 t\}}(t)
$$

whenever $\left|x-x^{\prime}\right|<\frac{1}{2}|x-y|$. Therefore,

$$
\begin{aligned}
\left|K(x, y)-T_{x^{\prime}} K(x, y)\right| & \leq \int_{0}^{\infty}\left|l_{t}(x, y)-T_{x^{\prime}} l_{t}(x, y)\right| \frac{d t}{t} \\
& \leq C\left|x-x^{\prime}\right|^{N+1} \int_{|x-y| / 4}^{\infty} \frac{\omega_{t}(x)}{t^{N+n+1}} \frac{d t}{t} \\
& =C\left|x-x^{\prime}\right|^{N+1} \int_{|x-y| / 4}^{\infty} \frac{\omega(B(x, t))}{t^{N+2 n+1}} \frac{d t}{t}
\end{aligned}
$$

Take $\bar{p}_{0}>p_{0}$ such that $N+I-\left(n \bar{p}_{0}-n\right)>0$ and $\omega \in A_{\bar{p}_{0}}$. Then

$$
\omega(B(x, t)) \leq\left(\frac{t}{\left|x-x^{\prime}\right|}\right)^{n \tilde{p}_{0}} \omega\left(B\left(x,\left|x-x^{\prime}\right|\right)\right)=\frac{t^{n \tilde{p}_{0}}}{\left|x-x^{\prime}\right|^{n \tilde{p}_{0}-n_{2}}} \omega_{\left|x ;-x^{\prime}\right|}(x),
$$

and we obtain

$$
\begin{aligned}
& \mid K(x, y)-T_{x^{\prime}} K(x, y) \mid \\
& \leq C\left|x-x^{\prime}\right|^{N-\left(n \bar{p}_{0}-n\right)+1} \omega_{\left|x-x^{\prime}\right|}(x) \int_{|x-y| / 4}^{\infty} \frac{t^{n \bar{p}_{0}}}{t^{N+2 n+1}} \frac{d t}{t} \\
& \quad=C \omega_{\left|x-x^{\prime}\right|}(x)\left|x-x^{\prime}\right|^{N+1-\left(n \bar{p}_{0}-n\right)} \frac{1}{|x-y|^{n+N+1-\left(n \bar{p}_{0}-n\right)}}
\end{aligned}
$$

whenever $\left|x-x^{\prime}\right|<\frac{1}{2}|x-y|$, where $\varepsilon=N+1-\left(n \bar{p}_{0}-n\right)>0$.
The same argument as above can be applied to the estimate of $\mid K(x, y)$ $T_{y^{\prime}} K(x, y) \mid$. This proves that the kernel of $T_{b}$ satisfies the second $\omega$-standard estimate (1.2) for $\varepsilon=N+1-\left(n \bar{p}_{0}-n\right)$.

We check the boundedness of $T_{b}: H^{p^{\prime}}(\omega) \rightarrow L^{p^{\prime}}\left(\omega^{1-p^{\prime}}\right)$ as follows. For $g \in L^{p}(\omega)$, we have

$$
\begin{aligned}
\left|\int T_{b} f(x) \cdot g(x) d x\right| \\
=\left|\int_{0}^{\infty} \int_{\mathbb{R}^{n}} Q_{t}(g) Q_{t}(b) P_{t}(f) \frac{d x d t}{t}\right| \\
\leq\left(\int\left(\int_{0}^{\infty}\left|Q_{t} g\right|^{2} \frac{d t}{t}\right)^{p / 2} \omega(x) d x\right)^{1 / p} \\
\times\left(\int\left(\int_{0}^{\infty}\left|Q_{t} b\right|^{2}\left|P_{t} f\right|^{2} \frac{d t}{t}\right)^{p^{\prime} / 2} \omega(x)^{1-p^{\prime}} d x\right)^{1 / p^{\prime}} \\
\leq C\|g\|_{L^{p}(\omega)}\left(\int\left(\int_{0}^{\infty}\left|Q_{t} b\right|^{2}\left|P_{t} f\right|^{2} \frac{d t}{t}\right)^{p^{\prime} / 2} \omega(x)^{1-p^{\prime}} d x\right)^{1 / p^{\prime}} .
\end{aligned}
$$

Now

$$
\begin{aligned}
\int\left(\int_{0}^{\infty}\left|Q_{t} b\right|^{2}\left|P_{t} f\right|^{2} \frac{d t}{t}\right)^{p^{\prime} / 2} \omega(x)^{1-p^{\prime}} d x \\
=\int\left(\int_{0}^{\infty}\left|Q_{t} b\right|^{p^{\prime}}\left|P_{t} f\right|^{p^{\prime}} g_{t}(x) \frac{d t}{t}\right) \omega(x)^{1-p^{\prime}} d x
\end{aligned}
$$

for some $g_{t} \geq 0$ with $\int_{0}^{\infty}\left|g_{t}(x)\right|^{r} \frac{d t}{t_{t}}=1$ for a.e. $x \in \mathbb{R}^{n}$, where $1 / r+p^{\prime} / 2$ $=1$.

CLAIM. $\left|Q_{t} b\right|^{p^{\prime}} g_{t}(x) \omega(x)^{1-p^{\prime}} \frac{d x d t}{t}$ is an $\omega$-Carleson measure.

Proof. Because $\omega^{1-p^{\prime}} \in A_{p^{\prime}}$, for any interval $I$, we have

$$
\begin{aligned}
\int_{0}^{|\lambda|^{1 / n}} \int_{I}\left|Q_{t} b\right|^{p^{\prime}} & g_{t}(x) \omega(x)^{1-p^{\prime}} \frac{d t d x}{t} \\
& =\int_{0}^{|I|^{1 / n}} \int_{I}\left|Q_{t}\left(\left(b-P_{I} b\right) \chi_{I^{*}}\right)\right|^{p^{\prime}} g_{t}(x) \frac{d t}{t} \omega(x)^{1-p^{\prime}} d x \\
& \leq \int\left(\int_{0}^{\infty}\left|Q_{t}\left(\left(b-P_{I} b\right) \chi_{I^{*}}\right)\right|^{2} \frac{d t}{t}\right)^{p^{\prime} / 2} \omega(x)^{1-p^{\prime}} d x
\end{aligned}
$$




$$
\leq \int_{I^{*}}\left|b-P_{I} b\right|^{p^{\prime}} \omega(x)^{1-p^{\prime}} d x \leq C \omega(I)\|b\|_{*}^{p^{\prime}} .
$$

Therefore,

$$
\begin{aligned}
\int\left(\int_{0}^{\infty}\left|Q_{t} b\right|^{2}\left|P_{t} f\right|^{2} \frac{d t}{t}\right)^{p^{\prime} / 2} \omega(x)^{1-p^{\prime}} d x \\
\quad=\iint\left|P_{t} f\right|^{p^{\prime}}\left|Q_{t} b\right|^{p^{\prime}} g_{t}(x) \omega(x)^{1-p^{\prime}} \frac{d x d t}{t} \\
\leq C\|b\|_{*}^{p^{\prime}} \int f^{* p^{\prime}} \omega(x) d x=C\|b\|_{*}^{p^{\prime}}\|f\|_{H p^{\prime}(\omega)}^{p^{\prime}} .
\end{aligned}
$$

where $f^{*}(x)=\sup _{|y-x|<t}\left|P_{t} f(y)\right|$. Consequently,

$$
\left|\int T_{b} f(x) \cdot g(x) d x\right| \leq C\|g\|_{L^{p}(\omega)}\|f\|_{H^{p^{\prime}}(\omega)}\|b\|_{*} .
$$

This proves that $T_{b}: H^{p^{\prime}}(\omega) \rightarrow L^{p^{\prime}}\left(\omega^{1-p^{\prime}}\right)$.

Unlike the case in [3] and [4], we cannot obtain the same estimate for $T_{b}^{t}$ by duality. Therefore we take another approach.

LEMMA 3.2. Let $T$ be a continuous linear operator from test functions to distributions that is associated with a kernel $K(x, y)$ which satisfies the second $\omega$-standard estimate (1.2). If $T: H^{q}(\omega) \rightarrow L^{q}\left(\omega^{1-q}\right)$ for some $1 \leq$ $q<\infty$, then $T^{t}: L^{\infty} \rightarrow B M O_{\omega}$.

Proof. For any $f \in \mathcal{S}$ (Schwartz class), and any interval $I$, we can write

$$
f=f \chi_{I^{*}}+f \chi_{I^{* c}}=f_{1}+f_{2}, \quad \text { where } I^{*}=4 I .
$$

We consider $T^{t} f_{2}$ first. Because $T$ is associated with the kernel $K(x, y)$,

$$
T^{t} f_{2}(y)=\int K(x, y) f_{2}(x) d x .
$$

Take $y_{0} \in 2 I \backslash \frac{3}{2} I$, let $P f_{2}(y)=\int T_{y_{0}} K(x, y) f_{2}(x) d x$, so $P f_{2}(y)$ is a polynomial of degree $\leq N$. Take $p>\max (2, q)$ such that $\omega \in A_{p}$. Then

$$
\begin{aligned}
\int_{I} \mid T^{t} f_{2}(y) & -\left.P f_{2}(y)\right|^{p^{\prime}} \omega^{1-p^{\prime}}(y) d y \\
& \leq \int_{I}\left(\int\left|K(x, y)-T_{y_{0}} K(x, y)\right| f_{2}(x) d x\right)^{p^{\prime}} \omega(y)^{1-p^{\prime}} d y \\
& \leq \int_{I}\left(\int_{I^{* c}} \frac{C \omega_{\left|y-y_{0}\right|}(y)\left|y-y_{0}\right|^{\varepsilon}}{|x-y|^{n+\varepsilon}}\left|f_{2}(x)\right| d x\right)^{p^{\prime}} \omega(y)^{1-p^{\prime}} d y \\
& \leq \int_{I}\left(C \omega_{\left|y-y_{0}\right|}(y)\|f\|_{\infty}\right)^{p^{\prime}} \omega(y)^{1-p^{\prime}} d y
\end{aligned}
$$

$$
\begin{aligned}
& \leq C\|f\|_{\infty}^{p^{\prime}} \int_{I}\left(\frac{\omega(I)}{|I|}\right)^{p^{\prime}} \omega(y)^{1-p^{\prime}} d y \\
& =C\|f\|_{\infty}^{p^{\prime}} \frac{1}{|I|^{p^{\prime}}}\left(\int_{I} \omega(y)^{1-p^{\prime}} d y\right)\left(\int_{I} \omega(y) d y\right)^{p^{\prime}} .
\end{aligned}
$$

Because $\omega \in A_{p}$,

$$
\begin{aligned}
& \left(\frac{1}{\omega(I)} \int_{I}\left|T^{t} f_{2}(y)-P f_{2}(y)\right|^{p^{\prime}} \omega(y)^{1-p^{\prime}} d y\right)^{1 / p^{\prime}} \\
& \quad \leq C\|f\|_{\infty}\left(\frac{1}{|I|} \int_{I} \omega d y\right)^{1-1 / p^{\prime}}\left(\frac{1}{|I|} \int_{I} \omega^{1-p^{\prime}} d y\right)^{1 / p^{\prime}} \leq C\|f\|_{\infty} .
\end{aligned}
$$

For $T^{t} f_{1}$, we have

$$
\begin{aligned}
& \left(\frac{1}{\omega(I)} \int_{I}\left|T^{t} f_{1}-P_{I} T^{t} f_{1}\right|^{p^{\prime}} \omega^{1-p^{\prime}} d x\right)^{1 / p^{\prime}} \\
& \quad=\sup _{\substack{\text { supp } a \subset I \\
x^{k} a d x=0, k=0,1, \ldots, N}}\left|\int\left(T^{t} f_{1}\right) a d x\right| \\
& \quad=\sup _{a\left(\omega(I)^{-1} \int_{I}|a|^{p} \omega d x\right)^{1 / p} \leq \omega(I)^{-1}}\left|\int f_{1} T a d x\right| \leq \sup _{a \text { a }(1, p) \text {-atom }} \int_{I^{*}}|T a| d x \cdot\|f\|_{\infty} .
\end{aligned}
$$

Now for $a$ a $(1, p)$-atom, we have

$$
\begin{aligned}
\int_{I^{*}}|T a| d x & \leq\left(\int_{I^{*}}|T a|^{q} \omega^{1-q} d x\right)^{1 / q}\left(\int_{I^{*}} \omega d x\right)^{1 / q^{\prime}} \\
& \leq C \omega(I)^{1 / q^{\prime}}\left(\int|T a|^{q} \omega^{1-q} d x\right)^{1 / q} \leq C \omega(I)^{1 / q^{\prime}}\|a\|_{H^{\alpha}(\omega)} .
\end{aligned}
$$

Following the same method as in [5, Chapter 2, §3], we get

$$
\|a\|_{H^{q}(\omega)} \leq C \omega(I)^{-1 / q^{\prime}}
$$

$(C$ does not depend on $a$ ). Thus

$$
\left(\frac{1}{\omega(I)} \int_{I}\left|T^{t} f_{1}-P_{I} T^{t} f_{1}\right|^{p^{\prime}} \omega^{1-p^{\prime}} d x\right)^{1 / p^{\prime}} \leq C\|f\|_{\infty} .
$$

Combining the two parts, we have proved

$$
\left\|T^{t} f\right\|_{B M O_{\omega}} \leq C\|f\|_{\infty}
$$

This means $T^{t}: L^{\infty} \rightarrow B M O_{\omega}$. 
As an immediate consequence, $T_{b}^{t}: L^{\infty} \rightarrow B M O_{\omega}$. By duality, we have $T_{b}: H^{1}(\omega) \rightarrow L^{\mathrm{J}}$

LEMMA 3.3. Let $T$ be a continuous linear operator from test functions to distributions that is associated with a kernel $K(x, y)$ which satisfies the second $\omega$-standard estimate (1.2). If $T: L^{\infty} \rightarrow B M O_{\omega}$, then $T: H^{1}(\omega) \rightarrow L^{1}$.

The proof of this lemma is almost the same as in [7, pp. 49-51]. For completeness, we present it here.

Proof. We need only estimate $\int|T a| d x$ for a a $(1, \infty)$-atom. Suppose $a$ is a $(1, \infty)$-atom associated with an interval $Q$, and $\bar{Q}=4 Q$. Then

$$
\int_{\bar{Q}^{c}}|T a| d x=\int_{\bar{Q}^{\circ}}\left|\int_{Q} K(x, y) a(y) d y\right| d x .
$$

Because $\int x^{k} a d x=0$ for $k=0,1, \ldots, N$, it follows that, for any $y_{0}$ in $2 Q \backslash \frac{3}{2} Q$

$$
\begin{aligned}
\int_{\bar{Q}^{c}}|T a| d x & =\int_{\bar{Q}^{c}}\left|\int_{Q}\left(K(x, y)-T_{y_{0}} K(x, y)\right) a(y) d y\right| d x \\
& \leq C \int_{\bar{Q}^{c}} \int_{Q} \frac{\omega_{\left|y-y_{0}\right|}(y)\left|y-y_{0}\right|^{\varepsilon}}{|x-y|^{n+\varepsilon}}|a(y)| d y d x \leq C,
\end{aligned}
$$

where $C$ does not depend on $a$

Let $Q^{\prime}$ be a cube of the same size as $\bar{Q}$, and such that $Q^{\prime}$ and $\bar{Q}$ have a face in common. Because $T: L^{\infty} \rightarrow B M O_{\omega},\|T a\|_{B M O_{\omega}} \leq C / \omega(Q)$. By the above calculation,

$$
\int_{Q^{\prime}}|T a| d x \leq C
$$

$$
\begin{aligned}
& \text { Now, } \\
& \frac{1}{\omega(\bar{Q})} \int_{\bar{Q}}|T a| d x \\
& \leq \frac{1}{\omega(\bar{Q})} \int_{\bar{Q}}\left|T a-P_{\bar{Q}} T a\right| d x+\frac{1}{\omega(\bar{Q})} \int_{\bar{Q}}\left|P_{\bar{Q}} T a\right| d x \\
& \leq\left(\frac{1}{\omega(\bar{Q})} \int_{\bar{Q}}\left|T a-P_{\bar{Q}} T a\right|^{p^{\prime}} \omega^{1-p^{\prime}} d x\right)^{1 / p^{\prime}}+\frac{1}{\omega(\bar{Q})} \int_{\bar{Q}}\left|P_{\bar{Q}} T a\right| d x \\
& \leq C\|a\|_{\infty}+\frac{1}{\omega(\bar{Q})} \int_{\bar{Q}}\left|P_{\bar{Q}} T a\right| d x \\
& \leq C \omega(Q)^{-1}+\frac{1}{\omega(\bar{Q})} \int_{\bar{Q}}\left|P_{\bar{Q}} T a\right| d x,
\end{aligned}
$$

and

$$
\begin{aligned}
& \int_{\bar{Q}}\left|P_{\bar{Q}} T a\right| d x \\
& \quad \leq \int_{\bar{Q}}\left|P_{\bar{Q}} T a-P_{Q^{\prime}} T a\right| d x+\int_{\bar{Q}}\left|P_{Q^{\prime}} T a\right| d x \\
& \quad \leq \int_{\overline{\bar{Q}}}\left|P_{Q^{\prime}}\left(T a-P_{\overline{\bar{Q}}} T^{\prime} a\right)\right| d x+\int_{\overline{\bar{Q}}}\left|P_{\bar{Q}}\left(T a-P_{\overline{\bar{Q}}} T a\right)\right| d x+\int_{\overline{\bar{Q}}}\left|P_{Q^{\prime}} T a\right| d x
\end{aligned}
$$

where $\overline{\bar{Q}}$ is a cube containing $\bar{Q} \cup Q^{\prime}$ and with size comparable to $Q$. (For example, $|\overline{\bar{Q}}| \leq 10^{n}|Q|$.) Because

$$
\int_{\bar{Q}}\left|P_{Q} f\right| d x \leq C \int_{Q}|f| d x
$$

for any $\bar{Q} \supset Q$ and $|\bar{Q}| \leq 2^{n}|Q|$,

$$
\int_{\bar{Q}}\left|P_{\bar{Q}} T a\right| d x
$$

$$
\begin{aligned}
& \leq C\left(\int_{\overline{\bar{Q}}}\left|T a-P_{\overline{\bar{Q}}} T a\right| d x+\int_{\overline{\bar{Q}}}\left|T a-P_{\overline{\bar{Q}}} T a\right| d x+\int_{Q^{\prime}}|T a| d x\right) \\
& \leq C\|a\|_{\infty} \omega(\overline{\bar{Q}})+C \leq C .
\end{aligned}
$$

Therefore,

$$
\frac{1}{\omega(\bar{Q})} \int_{\bar{Q}}|T a| d x \leq C \omega(Q)^{-1}+C \frac{1}{\omega(\bar{Q})},
$$

and then $\int_{\bar{Q}}|T a| d x \leq C$, which implies

$$
\int|T a| d x \leq \int_{\bar{Q}}|T a| d x+\int_{\bar{Q}^{c}}|T a| d x \leq C .
$$

This proves

$$
T: H I^{1}(\omega) \rightarrow L^{1}
$$

Up to now, we have proved that $T_{b}: H^{1}(\omega) \rightarrow L^{1}$ and $T_{b}^{t}: H^{1}(\omega) \rightarrow$ $L^{1}$. Take $\phi, \psi$ as in Lemma 3.1 such that $\int_{0}^{\infty} Q_{t}^{2} \frac{d t}{t}=I, \int \phi d x=1$, and $b=T 1, b_{1}=T^{t} 1$. Then $M=T-T_{b}-T_{b_{1}}^{t}$ satisfies $M 1=M^{t} 1=0$ and all the hypotheses of Theorem 2.1 except the first $\omega$-standard estimate (1.1). Therefore, it suffices to prove the sufficiency of Theorem 2.1 in the case 
where $T 1=0$, and $T^{t} 1=0$. As in [3] and [4], we can write

$$
\begin{aligned}
T & =\left(\int_{0}^{\infty} Q_{s}^{2} \frac{d s}{s}\right) T\left(\int_{0}^{\infty} Q_{t}^{2} \frac{d t}{t}\right) \\
& =\int_{0}^{\infty} \int_{0}^{\infty} Q_{s}\left(Q_{s} T Q_{t}\right) Q_{t} \frac{d t}{t} \frac{d s}{s} .
\end{aligned}
$$

The fact that $T: H^{1}(\omega) \rightarrow L^{1}$ now follows from the following two lemmas.

LEMMA 3.4. Same notations and assumptions as in Theorem 2.1 and Lemma 3.1. Assume also that $\phi, \psi$ satisfy the above hypotheses, and $T 1=0$, $T^{t} 1=0$. Then the kernel $K_{s, t}(x, y)$ of $Q_{s} T Q_{t}$ satisfies:

(1) for $0 \leq s \leq t$

$$
\left|K_{s, t}(x, y)\right| \leq\left(\frac{s}{t}\right)^{\varepsilon} \frac{t^{\varepsilon}}{(|x-y|+t)^{n+\varepsilon}} \omega_{s}(x),
$$

(2) for $0 \leq t \leq s$,

$$
\left|K_{s, t}(x, y)\right| \leq\left(\frac{t}{s}\right)^{\varepsilon} \frac{s^{\varepsilon}}{(|x-y|)^{n-\varepsilon}} \omega_{t}(x) .
$$

The proof of this lemma is the same as that of [3, Lemma 2.3], so we omit it.

LEMMA 3.5. If $K_{s, t}(x, y)$ satisfies the estimates in Lemma 3.4 and $f \in$ $H^{p^{\prime}}(\omega), g \in L^{p}(\omega)$, then

$$
\begin{aligned}
\int_{0}^{\infty} \int_{0}^{\infty} \int_{\mathbb{R}^{n}} \int_{\mathbb{R}^{n}}\left|Q_{s} g(x)\left\|K_{s, t}(x, y)\right\| Q_{t} f(y)\right| d x d y & \frac{d s}{s} \frac{d t}{t} \\
& \leq C\|f\|_{H^{p^{\prime}}(\omega)}\|g\|_{L^{p}(\omega)} .
\end{aligned}
$$

Proof. By assumption, the integral is controlled by

(3.6) $\quad C \iint_{s<t} \int_{\mathbb{R}^{2 n}} \int_{Q_{s}} g(x) \mid \omega_{s}(x)\left(\frac{s}{t}\right)^{\varepsilon}$

$$
\times \frac{t^{\varepsilon}}{(|x-y|+t)^{n+\varepsilon}}\left|Q_{t} f(y)\right| d x d y \frac{d s}{s} \frac{d t}{t}
$$

plus an analogous term for $s>t$. Setting

$$
P_{t}^{\varepsilon} h(x)=\int_{\mathbb{R}^{n}} \frac{t^{\varepsilon}}{(|x-y|+t)^{n+\varepsilon}} h(y) d y,
$$

we rewrite (3.6) and dominate it by

$$
C \int_{0}^{\infty} \int_{\mathbb{R}^{n}} \int_{0}^{t}\left|Q_{s} g(x)\right| \omega_{s}(x)\left(\frac{s}{t}\right)^{\varepsilon} P_{t}^{\varepsilon}\left(\left|Q_{t} f\right|\right)(x) \frac{d s}{s} \frac{d x d t}{t}
$$

$$
\begin{aligned}
& =C \int_{0}^{\infty} \int_{\mathbb{R}^{n}}\left(\int_{0}^{t}\left|Q_{s} g(x)\right| \frac{1}{s^{n}} \int_{|z-x|<s} \omega(z) d z\left(\frac{s}{t}\right)^{\varepsilon} \frac{d s}{s}\right) P_{t}^{\varepsilon}\left(\left|Q_{t} f\right|\right)(x) \frac{d x d t}{t} \\
& \leq C \int_{0}^{\infty} \int_{\mathbb{R}^{n}} \omega(z) \int_{0}^{t}\left(\frac{s}{t}\right)^{\varepsilon} \int_{|x-z|<s}\left|Q_{s} g(x)\right| \\
& \times \frac{s}{(s+|z-x|)^{n+1}} P_{t}^{\varepsilon}\left(\left|Q_{t} f\right|\right)(x) d x \frac{d s}{s} \frac{d z d t}{t} \\
& \leq C \int_{0}^{\infty} \int_{\mathbb{R}^{n}} \omega(z)\left(\int_{0}^{t}\left(\frac{s}{t}\right)^{\varepsilon} \frac{d s}{s}\right) \\
& \times\left(\int_{|x-z|<s} \frac{s}{(s+|z-x|)^{n+1}}\left|Q_{s} g(x)\right|^{2} d x\right)^{1 / 2} \\
& \times\left(\int_{|x-z|<t} \frac{s}{(s+|z-x|)^{n+1}}\left(P_{t}^{\varepsilon}\left(\left|Q_{t} f\right|\right)(x)\right)^{2} d x\right)^{1 / 2} \frac{d z d t}{t} \\
& \leq C \int_{0}^{\infty} \int_{\mathbb{R}^{n}} \omega(z) \int_{0}^{t}\left(\frac{s}{t}\right)^{\varepsilon} Q_{1, s}(|g|)(z) \frac{d s}{s} \cdot Q_{2, t}(|f|)(z) \frac{d z d t}{t} \\
& \leq C \int_{\mathbb{R}^{n}} \omega(z)\left(\int_{0}^{\infty}\left(\int_{0}^{t}\left(\frac{s}{t}\right)^{\varepsilon} Q_{1, s}(|g|)(z) \frac{d s}{s}\right)^{2} \frac{d t}{t}\right)^{1 / 2} \\
& \times\left(\int_{0}^{\infty} Q_{2, t}^{2}(|f|)(z) \frac{d t}{t}\right)^{1 / 2} d z \\
& \leq C\left(\int_{\mathbb{R}^{n}} \omega(z)\left(\int_{0}^{\infty}\left(\int_{0}^{t}\left(\frac{s}{t}\right)^{\varepsilon} Q_{1, s}(|g|)(z) \frac{d s}{s}\right)^{2} \frac{d t}{t}\right)^{p / 2} d z\right)^{1 / p} \\
& \times\left(\int_{\mathbb{R}^{n}} \omega(z)\left(\int_{0}^{\infty} Q_{2, t}^{2}(|f|)(z) \frac{d t}{t}\right)^{p^{\prime} / 2} d z\right)^{1 / p^{\prime}} \\
& \leq C\left(\int_{\mathbb{R}^{2}} \omega(z)\left(\int_{0}^{\infty} \int_{0}^{t}\left(\frac{s}{t}\right)^{\varepsilon} Q_{1, s}^{2}(|g|)(z) \frac{d s}{s} \frac{d t}{t}\right)^{p / 2} d z\right)^{1 / p}\|f\|_{H^{p^{\prime}}(\omega)} \\
& \leq C\left(\int_{\mathbb{R}^{r t}} \omega(z)\left(\int_{0}^{\infty} Q_{1, s}^{2}(|g|)(z) \frac{d s}{s}\right)^{p / 2} d z\right)^{1 / p}\|f\|_{H^{p^{\prime}}(\omega)} \\
& \leq C\left(\int_{\mathbb{R}^{n}}\left|g^{*}\right|^{p} \omega(z) d z\right)^{1 / p}\|f\|_{H^{p^{\prime}}(\omega)} \leq C\|g\|_{L^{p}(\omega)}\|f\|_{H^{p^{\prime}}(\omega)}
\end{aligned}
$$


because $\omega \in A_{p}$. The seventh and ninth inequalities are true according to the results in [2]. Now we have proved the sufficiency of Theorem 2.1. By Lemma 3.3 and duality, we obtain the sufficiency of Corollary 2.2 .

The necessity of Theorem 2.1 and Corollary 2.2 is proved by using Lemma 3.3 and duality. Because $T: H^{1}(\omega) \rightarrow L^{1}$, by duality, we have $T^{t}: L^{\infty} \rightarrow B M O_{\omega}$, and then by Lemma 3.3 , we have $T^{t}: H^{1}(\omega) \rightarrow L^{1}$, and so $T: L^{\infty} \rightarrow B M O_{\omega}$. Therefore, $T 1 \in B M O_{\omega}$ and $T^{t} 1 \in B M O_{\omega}$.

The fact that $T$ satisfies the $\omega-W B P$ is an easy consequence of $T$ : $H^{1}(\omega) \rightarrow L^{1}$

4. The Calderón-Zygmund theory for $\omega$-CZO. The interpolation result in [9, Chapter $2, \S 3]$ enables us to establish the Calderón-Zygmund theory for $\omega$-CZO.

THEOREM 4.1. Suppose that $\omega \in A_{\infty}$. Let $T$ be a continuous linear operator from test functions to distributions that is associated with an $\omega$-standard kernel. Suppose further that $T: H^{1}(\omega) \rightarrow L^{1}$. Then $T: H^{p}(\omega) \rightarrow\left(H^{p^{\prime}}(\omega)\right)^{*}$ for $1<p<\infty$, where $1 / p+1 / p^{\prime}=1$ and $\left(H^{p^{\prime}}(\omega)\right)^{*}$ is the dual space of $H^{p^{\prime}}(\omega)$. Precisely, we have

$$
\int\left(\sum_{2 Y \ni x}\left((T f)_{I} \frac{|I|}{\omega(I)}\right)^{2}|I|^{-1}\right)^{p / 2} \omega(x) d x \leq C\|f\|_{H^{p}(\omega)}^{p}
$$

where $(T f)_{I}=\int T f \psi_{I} d x$ with the notations as in [9, Chapter 2].

Proof. By Corollary 2.2, we have $T: L^{\infty} \rightarrow B M O_{\omega}$. Applying Tom Wolff's theorem [8] and the interpolation result in [9], we obtain $\left[H^{1}(\omega), L^{\infty}\right]_{\theta}=H^{p}(\omega)$, where $1 / p=1-\theta$. By the duality theorem and the reiteration theorem [1] and Tom Wolff's theorem [8],

$$
\begin{aligned}
{\left[L^{1}, B M O_{\omega}\right]^{\theta} } & \subset\left[\left(L^{\infty}\right)^{*}, B M O_{\omega}\right]^{\theta}=\left[\left(L^{\infty}\right)^{*},\left(H^{1}(\omega)\right)^{*}\right]^{\theta} \\
& =\left(H^{r}(\omega)\right)^{*}=\left[L^{1}, B M O_{\omega}\right]_{\theta},
\end{aligned}
$$

where $1 / r=\theta$. Therefore we have $T: H^{p}(\omega) \rightarrow\left(H^{p^{\prime}}(\omega)\right)^{*}$, where $1<p<$ $\infty, 1 / p+1 / p^{\prime}=1$. (4.2) is a direct consequence of the duality result in [9].

\section{References}

[1] J. Bergh and J. Löfström, Interpolation Spaces, Springer, New York, 1976.

2] D. L. Burkholder, Martingale theory and harmonic analysis in Euclidean spaces, in: Proc, Sympos. Pure Math. 35, Part 2, Amer. Math. Soc., 1979, 283-301.

[3] R. Coifman, G. David, Y. Meyer and S. Semmes, w-Calderón-Zygmund operators, in: Lecture Notes in Math. 1384, Springer, 1989, 132-145.

[4] G. David and J.-L. Journé, A boundedness criterion for generalized CalderónZygmund operators, Ann. of Math, 120 (1984), 371-397.
[5] J. García-Cuerva, Weighted $H^{p}$-spaces, Dissertationes Math. 162 (1979).

6] García-Cuerva and J. L. Rubio de Francia, Weighted Norm Inequalities and Related Topics, North-Holland, 1985

[7] J.-L. Journé, Calderón-Zygmund Operators, Pseudo-Differential Operators and the Cauchy Integral of Calderón, Lecture Notes in Math. 994, Springer, 1983.

[8] T. H. Wolff, A note on interpolation spaces, in: Lecture Notes in Math. 908, Springer, 1981, 199-204.

[9] S. W u, A wavelet characterization for weighted Hardy spaces, Rev. Mat. Iberoamericana $8(1.992), 329-349$

DEPARTMENT OF MATHEMATICS

NORTHWESTERN UNIVERSITY

EVANSTON, IRLINOIS 60208

U.S.A. 